\title{
Short communication: Molecular characteristics, antimicrobial susceptibility, and pathogenicity of clinical Nocardia cyriacigeorgica isolates from an outbreak of bovine mastitis
}

\author{
Wei Chen, ${ }^{*}$ Yongxia Liu,† Herman W. Barkema, $\ddagger$ Jian Gao, ${ }^{*}$ Jeroen De Buck, $\ddagger$ John P. Kastelic, $\ddagger$ Gang Liu, ${ }^{*}$ \\ Tariq Ali, ${ }^{*}$ Muhammad Shahid, ${ }^{*}$ and Bo Han*1 \\ ${ }^{*}$ College of Veterinary Medicine, China Agricultural University, Yuan Ming Yuan West Road No. 2, Haidian District, Beijing 100193, P. R. China \\ †College of Veterinary Medicine, Shandong Agricultural University, Tai'an 271018, China \\ ‡Department of Production Animal Health, Faculty of Veterinary Medicine, University of Calgary, Calgary, AB, Canada, T2N 4N1
}

\begin{abstract}
The occurrence of nocardial mastitis, mostly in the context of outbreaks, has been reported in many countries. However, there is a paucity of reports regarding detailed characterization of Nocardia cyriacigeorgica from bovine mastitis. Thus, herein we report characteristics, antimicrobial susceptibility patterns, molecular identification, and pathogenicity of $N$. cyriacigeorgica isolated from an outbreak of clinical mastitis in a dairy herd in northern China. A total of $182(80.2 \%)$ lactating cows had clinical mastitis with severe inflammation and firmness of the udder, reduced milk production, and anorexia, with no apparent clinical response to common antibiotics. Out of 22 mastitic milk samples submitted to our laboratory, 12 N. cyriacigeorgica were isolated and characterized using standard microbiological analysis, 16S rRNA gene sequencing, random amplified polymorphic DNA PCR analysis, biochemical assays, and antibiotic susceptibility testing. Additionally, in vivo experiments were done to determine pathogenicity of these clinical mastitis isolates. All isolates were resistant to ampicillin, amoxicillin-clavulanic acid, ciprofloxacin, minocycline, rifampicin, and aminoglycosides (type VI pattern). Additionally, intramammary inoculation of mice with $N$. cyriacigeorgica caused chronic inflammatory changes, including hyperemia, edema, and infiltration of lymphocytes and neutrophils, as well as hyperplasia of lymph nodules in mammary glands. Therefore, we concluded that $N$. cyriacigeorgica was involved in the current outbreak of mastitis. To our best knowledge, this is the first report to characterize $N$. cyriacigeorgica isolated from cases of bovine mastitis in China.
\end{abstract}

Received February 2, 2017.

Accepted June 28, 2017.

${ }^{1}$ Corresponding author: hanbo@cau.edu.cn
Key words: bovine mastitis, Nocardia cyriacigeorgica, outbreak, antimicrobial resistance, pathogenicity

\section{Short Communication}

Multidrug-resistant Nocardia spp. have been reported to cause nocardiosis in humans and dairy cattle (e.g., Condas et al., 2013; Hashemi-Shahraki et al., 2015). Nocardia is a genus of aerobic and saprophytic actinomycetes, gram-positive, branching, filamentous, and modified acid-fast bacilli. These environmental pathogens are ubiquitous in air, soil, water, and decaying organic matter (Brown-Elliott et al., 2006).

Mastitis is the most common clinical manifestation of nocardial infections in livestock (Condas et al., 2013). Nocardia spp. are considered a causative agent of bovine mastitis, with occurrence reported in several countries (Cook and Holliman, 2004; Pisoni et al., 2008; Ribeiro et al., 2008). Clinically, nocardial mastitis is characterized by a suppurative or granulomatous inflammation of the mammary gland, with severe clinical manifestations, diffuse udder fibrosis and firmness, reduced milk production, and altered milk appearance, as well as systemic involvement, including anorexia and fever (Pisoni et al., 2008; Kogure et al., 2010; Condas et al., 2013). Nocardia spp. are usually resistant to most antimicrobials commonly used in veterinary medicine (e.g., gentamicin, amoxicillin-clavulanic acid, ampicillin, ciprofloxacin, kanamycin, and ceftriaxone), leading to chronic IMI resulting in drying up of the quarter and culling of the cow (Radostits et al., 2007). Interestingly, Nocardia spp. have a variety of antibiotic susceptibility patterns, which can be used for phenotypic identification (Brown-Elliott et al., 2006).

The most prevalent species of Nocardia associated with bovine mastitis are Nocardia farcinica, Nocardia asteroides, Nocardia nova, and Nocardia brasiliensis (Radostits et al., 2007; Condas et al., 2013). So far, apparently, only 1 report is available regarding isola- 
tion of $N$. cyriacigeorgica from bovine mastitis (Condas et al., 2013), whereas nocardiosis caused by $N$. cyriacigeorgica is more frequently reported in humans (Hashemi-Shahraki et al., 2015; Xiao et al., 2016). The aim of present study was to investigate the biochemical characteristics and phylogenetic relatedness using 16S rRNA gene sequencing, antimicrobial susceptibility patterns, and pathogenicity of $N$. cyriacigeorgica isolated from a severe outbreak of bovine mastitis.

In a commercial privately owned dairy herd located in Tangshan city, Hebei province, in the north of China, composed of 550 Holstein-Friesian cattle, including 227 lactating cows, an outbreak of severe clinical mastitis (CM) occurred June 11 to 18, 2013. The lactating cows were fed a TMR and were housed in a bedded-pack barn with inadequate bedding and a surface covered with wet manure. Before the outbreak, there was $10 \mathrm{~d}$ of continuous rain and daily peak ambient temperatures were 22 to $29^{\circ} \mathrm{C}$. Lactating cows were milked thrice daily by 4 milking staff at 0600,1400 , and $2000 \mathrm{~h}$ in a $2 \times$ 12 parallel milking parlor. The milking machine was inadequately maintained, with excessive vacuum pressure $(47.8 \mathrm{kPa})$. Both pre- and postmilking teat disinfection was done by dipping with $0.15 \%$ iodine solution. One towel was used for 2 cows. At drying off, all cows were given intramammary treatments with Orbenin EDC (Cloxacillin benzathine, Zoetis, Parsippany, NJ). The herd did not use any self-compounded drugs. During the outbreak, 22 quarter milk samples were aseptically collected from 22 cows with CM that had not recently received antibiotic treatment. Samples were shipped on ice to the mastitis laboratory at the College of Veterinary Medicine, China Agricultural University, Beijing.

Milk samples $(10 \mu \mathrm{L})$ were streaked onto tryptose soy agar (TSA; Aoboxing Bio-Tech, Beijing, China) supplemented with $5 \%$ defibrinated sheep blood (also known as sheep blood agar, SBA) using aseptic cotton swabs and incubated for 24 to $72 \mathrm{~h}$ at $37^{\circ} \mathrm{C}$. Nocardialike colonies were sub-cultured on SBA, Middlebrook 7H10 agar supplemented with $0.5 \%$ glycerol, and $10 \%$ Middlebrook ADC enrichment (7H10, Becton Dickinson and Company, Franklin Lakes, NJ) and Gause's synthetic agar (GSA, Land Bridge Technology Co., Ltd., Beijing, China). Staining properties were tested by Gram and Ziehl-Neelsen staining (Solarbio Science and Technology, Beijing, China). Spores and mycelium were investigated with a light microscope (Olympus, Tokyo, Japan) and a scanning electron microscope (Hitachi S-3400N, Tokyo, Japan).

Molecular identification of clinical isolates was done with 16S rRNA sequence analysis, using universal primers 27F (5'-AGAGTTTGATCCTGGCTCAG- $\left.3^{\prime}\right)$ and 1492R (5'-TACGGCTACCTTGTTACGACTT-3'), which were used for both amplification and sequenc- ing. Sequences were analyzed in the National Center for Biotechnology Information database using the Basic Local Alignment Search Tool (BLAST). The phylogenetic tree, based on 100 bootstrap replicates, was constructed by employing the neighbor joining method using MEGA 5.2 software (http://www.megasoftware .net), based on the $16 \mathrm{~S}$ rRNA gene sequences trimmed to 1,387 bp of clinical Nocardia isolates and some other Nocardia species ATCC strains downloaded from the National Center for Biotechnology Information (Figure 1). Genetic distances were calculated using a Kimura's 2-parameter model. Mycobacterium bovis ATCC BAA935 (CP009449.1) was used as an outgroup species.

Random amplified polymorphic DNA (RAPD) PCR analysis was performed with 12 N. cyriacigeorgica isolates using the RAPD primer RBA 15A 5'-AAGAGCCCGT-3' according to previously described protocols (Trivedi, 2015). Amplified DNA fragments were separated on a 1.5\% agarose gels, stained with ethidium bromide, and visualized by gel documentation system (Alpha Imager EC, San Leandro, CA). The similarity between strains was clustered by using the Dice coefficient and the unweighted pair group method with arithmetic averages (UPGMA) of the PFGE profiles (Liu et al., 2015). Growth of N. cyriacigeorgica isolates was assessed on duplicate TSA plates, incubated at 37 and $45^{\circ} \mathrm{C}$ for $5 \mathrm{~d}$. Biochemical tests were performed according to the manufacturer's instructions (http:// www.tianhe138.com/download5.aspx? InfoVer $=500 \&$ ClassID $=20 \& P I D=5$ ), which included hydrolysis of adenine, L-tyrosine, xanthine, hypoxanthine, casein, urea (Sigma, St. Louis, MO); utilization of citrate, esculin, rhamnose, sorbitol, L-arabinose, fructose, galactose, lactose, mannitol, inositol, xylose, glucose, sucrose, and acetamide; and production of catalase and nitrate reductase (Hangzhou Binhe Microorganism Reagent Co. Ltd., Hangzhou, China). In addition, adenine, hypoxanthine, L-tyrosine, xanthine, and casein decomposition were also performed (Gordon et al., 1974), and results were assessed after 7 and $14 \mathrm{~d}$. Negative controls, without Nocardia, were similarly processed. For the catalase test, a loop of colony was transferred to a drop of $3 \%$ $\mathrm{H}_{2} \mathrm{O}_{2}$; the reaction was recorded as positive if bubbles appeared immediately.

Antimicrobial susceptibility patterns of all isolates were determined against 15 antimicrobial agents using the MIC method (broth microdilution), according to CLSI M24-A2 (CLSI, 2011) recommendations. Antimicrobial agents included amoxicillin-clavulanic acid (5:1), amikacin, cefotaxime, ciprofloxacin, minocycline, sulfamethoxazole, tetracycline (BBI Life Sciences Corporation, Shanghai, China), imipenem, rifampicin, clarithromycin, and ceftiofur (Sigma), gentamicin, ampicillin, kanamycin (Amresco, Solon, OH), and pirlimy- 
cin (Yuduo Biological Technology Co., Ltd., Shanghai, China). In the absence of CLSI breakpoints for ampicillin, kanamycin, ceftiofur, pirlimycin, and tetracycline for Nocardia spp., CLSI M07-A9 recommendations for aerobic bacteria (CLSI, 2012) and VET01 recommendations for bacteria isolated from animals were used (CLSI, 2013). Staphylococcus aureus ATCC 29213 was used as the quality control strain.

Thirty-six pregnant 12-wk-old outbred ICR mice were purchased from Beijing Vital River Laboratory Animal Technology (Beijing, China) to determine pathogenicity of clinical $N$. cyriacigeorgica isolates. At $3 \mathrm{~d}$ postpartum, suckling mice were removed and the dams were innoculated. Mice were anaesthetized with tiletamine hydrochloride and zolazepam hydrochloride; $10 \mathrm{mg} / \mathrm{kg}$ injected i.m.; Zoletil 50, Virbac Laboratories, Carros, France), and inoculated with $2.5 \times 10^{4} \mathrm{cfu}$ of the mid-log phase $N$. cyriacigeorgica, placed in a $1-\mathrm{mL}$ sterile syringe and injected through a 32-gauge blunt needle into the nipples of the fourth and fifth left abdominal mammary glands. Intramammary inoculation was performed with the assistance of a bench magnifier $(3.0 \times)$. In negative control groups, mice were inoculated with sterile $0.1 M$ PBS ( $\mathrm{pH} 7.4$ ).

Clinical symptoms of challenged mice were scored as follows: $0=$ normal; $1=$ mildly messy fur; $2=$ depression and obvious messy fur; $3=$ cowered behavior and moderate depression; $4=$ serious depression with prostration; and $5=$ death. In both PBS and Nocardia-challenged groups, 18 mice were scored on $\mathrm{d} 1$, whereas $15,12,9$, and 6 mice were scored on $\mathrm{d} 3,5,9$, and 15 , respectively. On d 1, 3, 5, 9, and 15 after inoculation, 3 mice were euthanized using cervical dislocation, inoculated mammary glands were excised aseptically, and samples fixed in $10 \%$ formalin buffer and processed using standard histopathological methods. Histopathological scoring of the mammary gland of mice challenged with $N$. cyriacigeorgica and PBS

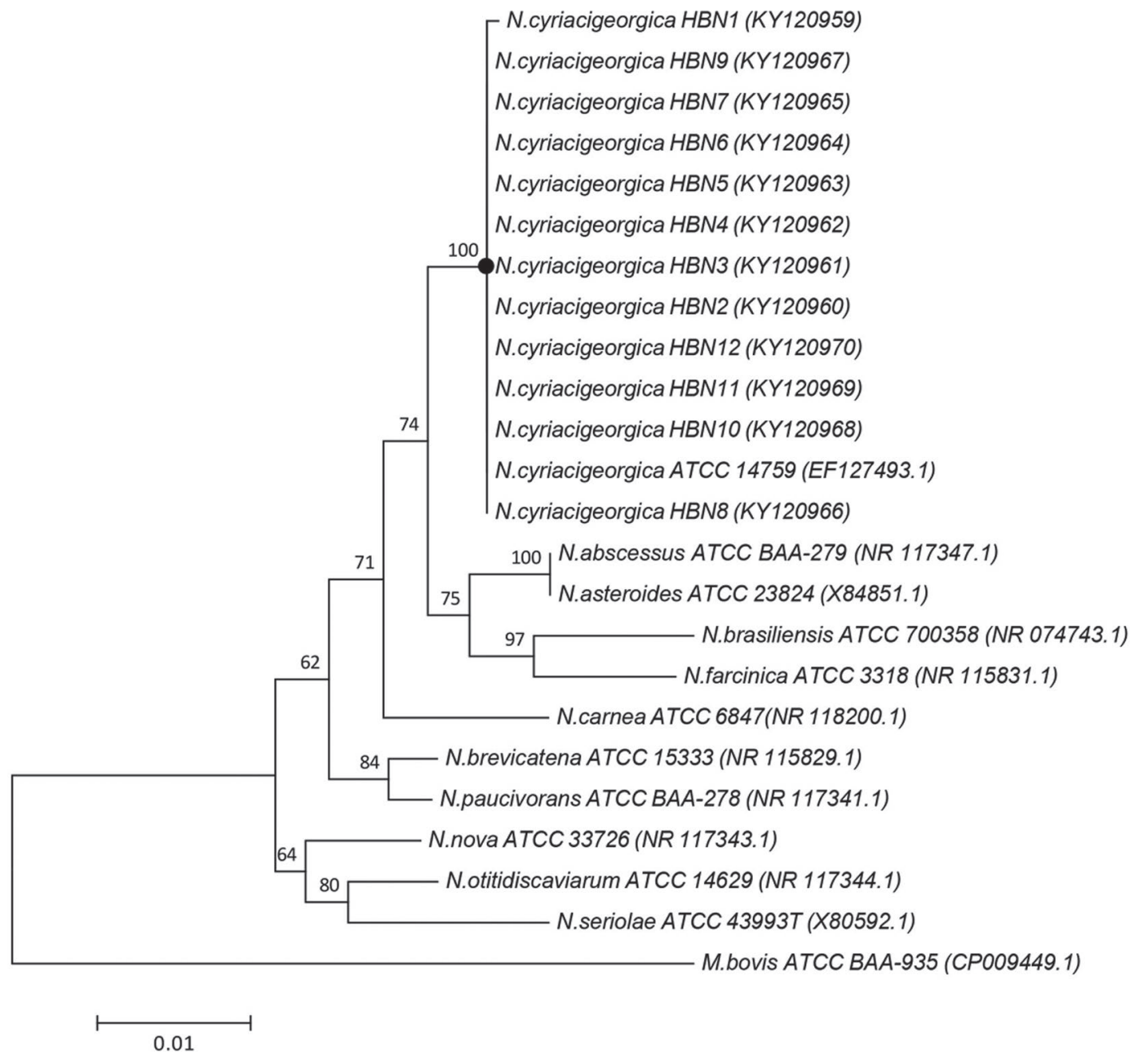

Figure 1. Phylogenetic tree of clinical Nocardia cyriacigeorgica isolates and other Nocardia ATCC strains, based on 16S rRNA gene sequencing of 1,387-bp amplicons. Bar indicates the percentage of sequence divergence. 
was performed according to lesion severity: $0=a b-$ sence of histopathological changes; mild, sub-moderate, moderate, and severe were scored as $1,2,3$, and 4 , respectively. Three mice were scored on the specific day both in PBS and Nocardia-challenged groups, with 3 pathological sections per mouse mammary gland. Data were represented as mean \pm standard deviation. Differences between $N$. cyriacigeorgica and PBS-challenged groups at the same time point were estimated using a Student's $t$-test in GraphPad Prism version 5.0 for Windows (GraphPad Software Inc., La Jolla, CA). $P<$ 0.05 and $P<0.01$ were regarded as statistically significant and highly significant, respectively.

The animal experiment was conducted in compliance with guidelines of the Beijing Municipality on the Review of Welfare and Ethics of Laboratory Animals, approved by the Beijing Municipality Administration Office of Laboratory Animals and under a protocol (CAU-AEC-2010-0603) approved by the China Agricultural University (Beijing, P.R. China) Animal Ethics Committee.

A total of 206 quarters, 81 rear quarters and 125 front quarters, of $182(80.2 \%)$ lactating cows were affected with CM. Of the 182 cows with CM, 61 (73\%) of 84 were first lactation heifers, $56(78 \%)$ of 72 were in second parity, and 65 (92\%) of 71 were cows with parity $\geq 3$. No CM cases were observed among dry cows and nonlactating heifers. Average milk production before the outbreak was $25 \mathrm{~L} / \mathrm{d}$, whereas after the outbreak, milk production decreased to $15 \mathrm{~L} / \mathrm{d}$. Bulk milk SCC was $\sim 450,000$ cells $/ \mathrm{mL}$ before the outbreak, but increased to 800,000 cells $/ \mathrm{mL}$ after the outbreak (milk from all affected cows was included in the bulk tank after treatment). During the CM outbreak, individual SCC of unaffected animals was not determined, but average SCC of those cows apparently did not increase.

Clinical manifestations in all affected cattle, as noted by the herd's veterinarian, consisted of severe inflammation of mammary glands, firmness of the udder tissues, reduced milk production along with abnormal consistency of milk, and systemic signs of anorexia and conjunctiva hyperemia. No increase was observed in the incidence of other health events. Once daily, cows with CM were given ceftiofur sodium (Shangqiu Huakang Animal Pharmaceutical Co., Ltd., Henan province, China) as an intramammary infusion and intramuscular injections of $4 \times 10^{6}$ IU of benzylpenicillin potassium (Beijing Cephal Pharmaceutical Corporation, Beijing, China) and 1 million IU of kanamycin sulfate (Beijing Lishida Pharmaceutical Co., Ltd., Beijing, China) until symptoms disappeared. However, many $\mathrm{CM}$ cases did not cure clinically, and $23(13 \%)$ of cows were eventually culled.
Pure growth of $N$. cyriacigeorgica was obtained on SBA plates for 12 of the 22 quarter milk samples. At least 3 types of bacterial colonies were observed on 5 SBA plates, indicating contamination, whereas 5 samples were culture negative. After $72 \mathrm{~h}$ incubation, $N$. cyriacigeorgica colonies showed a different appearance on SBA, Middlebrook 7H10, and GSA by visual and microscopic observation (Supplemental Figures S1A-S1F; https://doi.org/10.3168/jds.2017-12680). Nocardia cyriacigeorgica isolates were Gram-positive (Supplemental Figures S1G and S1H; https://doi.org/ 10.3168/jds.2017-12680), but were partially positive using Ziehl-Neelsen (Supplemental Figure S1I; https:/ / doi.org/10.3168/jds.2017-12680). When viewed with a scanning electron microscope, $N$. cyriacigeorgica appeared as spherical spores, filaments that can fragment into bacillary and coccoid forms on 7H10 agar (Supplemental Figures S2A and SB2; https://doi.org/10.3168/ jds.2017-12680), or Streptococcus-like mycelium on GSA agar (Supplemental Figure S2C; https://doi.org/ 10.3168/jds.2017-12680).

Based on BLAST analysis of $16 \mathrm{~S}$ rRNA gene sequencing, clinical isolates were closely related to $N$. cyriacigeorgica, $N$. asteroides, and $N$. abscessus (homologies of 99-100, 98-99, and 98-99\%, respectively). The phylogenetic tree, based on 16S rRNA gene sequence of 1,387-bp sized amplicons, is shown (Figure 1). The $N$. cyriacigeorgica isolates (HB1 to HB12) formed a cluster with N. cyriacigeorgica ATCC 14759 with a high bootstrap value of 100; furthermore, the same bootstrap value was also observed between $N$. abscessus ATCC BAA-279 and $N$. asteroides ATCC 23824. In addition, clustering of $N$. cyriacigeorgica formed a branch with the nearby clustering of $N$. abscessus ATCC BAA-279, $N$. asteroides ATCC 23824, N. brasiliensis ATCC 700358, and $N$. farcinica ATCC 3318, with a bootstrap value of 74 indicating a close relationship among them. The RAPD-PCR analysis of 12 isolates showed a percentage of similarity ranging from approximately 38 to $91 \%$ (Supplemental Figure S3; https://doi.org/10.3168/jds .2017-12680), indicating high variability of these $N$. cyriacigeorgica isolates.

All $N$. cyriacigeorgica isolates were able to grow at both 37 and $45^{\circ} \mathrm{C}$ on TSA plates. All isolates were strongly positive for catalase, utilization of acetamide, and reduction of nitrate to nitrite (Supplemental Table S1; https://doi.org/10.3168/jds.2017-12680). Results for esculin hydrolysis and acid formation from galactose and xylose fermentation on d 2 were inconclusive; however, obvious color changes were observed when incubation was extended to d 5. Additionally, isolates were negative for hydrolysis of adenine, L-tyrosine, xanthine, hypoxanthine, casein, and urea; and utilization of ci- 
trate, rhamnose, sorbitol, arabinose, fructose, lactose, mannitol, inositol, glucose, and sucrose (Supplemental Table S1; https://doi.org/10.3168/jds.2017-12680).

All clinical isolates were uniformly resistant to gentamicin, amoxicillin-clavulanic acid, clarithromycin, ciprofloxacin, minocycline, ampicillin, refampin, kanamycin, pirlimycin, and tetracycline. Additionally, they had high-level resistance to clarithromycin (MIC >128 $\mu \mathrm{g} / \mathrm{mL}$ ), amoxicillin-clavulanic acid (MIC $\geq 64 \mu \mathrm{g} /$ $\mathrm{mL}$ ), ampicillin (MIC $\geq 64 \mu \mathrm{g} / \mathrm{mL}$ ), rifampicin (MIC $>64 \mu \mathrm{g} / \mathrm{mL}$ ), kanamycin ( $\mathrm{MIC}>256 \mu \mathrm{g} / \mathrm{mL}$ ), pirlimycin $(\mathrm{MIC}>64 \mu \mathrm{g} / \mathrm{mL}$ ), and tetracycline $(\mathrm{MIC} \geq 32$ $\mu \mathrm{g} / \mathrm{mL})$. Also, all N. cyriacigeorgica isolates were susceptible to amikacin and sulfamethoxazole, whereas 10 isolates were susceptible to cefotaxime and imipenem. Finally, 3 isolates were susceptible to ceftiofur and 1 isolate was resistant to ceftiofur (Table 1).

Starting at $3 \mathrm{~d}$ after inoculation, mice inoculated with $N$. cyriacigeorgica increasingly had signs of messy fur and anorexia, were sluggish, and had slightly cowered behavior in comparison to control mice $(P<0.01$; Figure 2A). Inoculated mammary glands had no prominent visual signs; however, gross examination during autopsy revealed edema, hyperemia, and formation of nodules (diameter, 2-3 $\mathrm{mm}$ ) in mammary glands at d 5. Histopathologically, mammary epithelial cells had vacuolated degeneration on d 1 (Figure 2C). On d 3, necrosis and severe exfoliation of epithelial cells appeared in the mammary alveolar tissues (Figure 2D), with infiltration of inflammatory cells, edema, and congestion present in the mesenchymal tissues from $d$ 5. Furthermore, inflammatory cells and necrotic epithelial cells were also observed in mammary ducts and in atrophic alveoli (Figure 2E, 2F, and 2G). Additionally, inflammatory cells appeared in blood vessels in mammary gland and lymphoid tissues, and some mastocytes were detected in mammary glands on d 9. Degeneration and necrosis was observed in mammary epithelial cells (Figure 2G). The grossly apparent nodules were lymph nodes with swelling and hyperplasia (Figure $2 \mathrm{H}$ ), with germinal centers with abnormal inflammatory cells and vacuolation in the necrotic areas. Mean histopathological scores of mouse mammary glands are presented (Figure 2B). In comparison to the control group, histopathological changes were observed in the mammary gland of mice challenged with $N$. cyriacigeorgica $(P<0.01)$, including infiltration of inflammatory cells, necrosis of epithelial cells, and hyperplasia of lymph nodes (mentioned above).

In this study, 12 clinical $N$. cyriacigeorgica isolates from an outbreak of CM were characterized. Accurate identification of Nocardia spp. is necessary for clinical epidemiological studies. Bovine nocardial mastitis has been reported to be strongly associated with poor man-

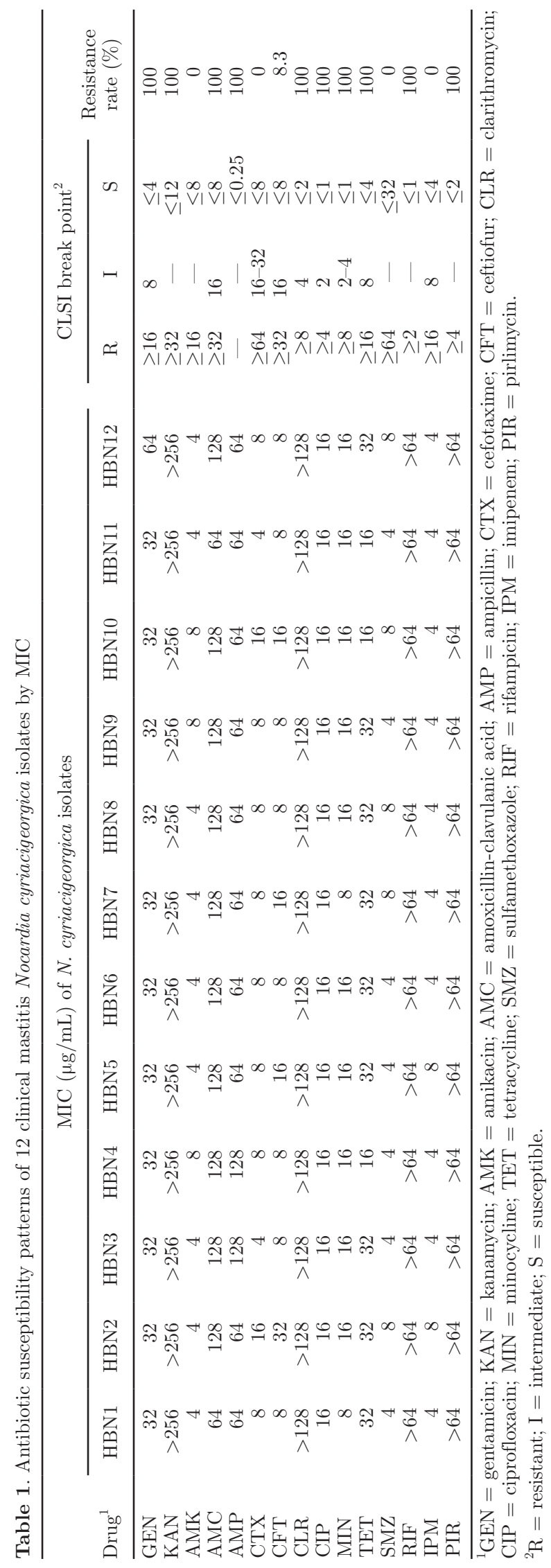


agement practices and unhygienic measures in dairy herds (Ribeiro et al., 2008; Ali et al., 2014). Factors including improper disinfection of teats before and after milking and intramammary infusions with contaminated instruments or pharmaceuticals predisposed to nocardiosis (Ollis et al., 1991; Cook and Holliman, 2004). The dairy herd in the present study had poor hygiene and insufficient milking management and mastitis control practices, with high isolation rates in
CM samples of other bacteria including Bacillus cereus (41\%), Escherichia coli (36\%), Staphylococcus chromogenes (23\%), and Streptococcus dysgalactiae (14\%). Unfortunately, samples from bedding and the milking parlor were not collected for culture, and because the farm stopped dairy activities shortly after the outbreak, no samples could be obtained retrospectively. Given the lack of similarity in RAPD-PCR analysis (Supplemental Figure S3; https://doi.org/10.3168/jds.2017-12680),
A

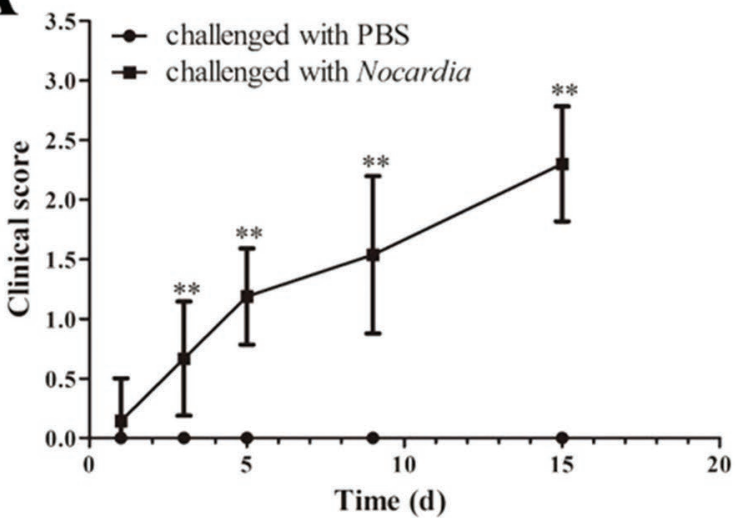

B

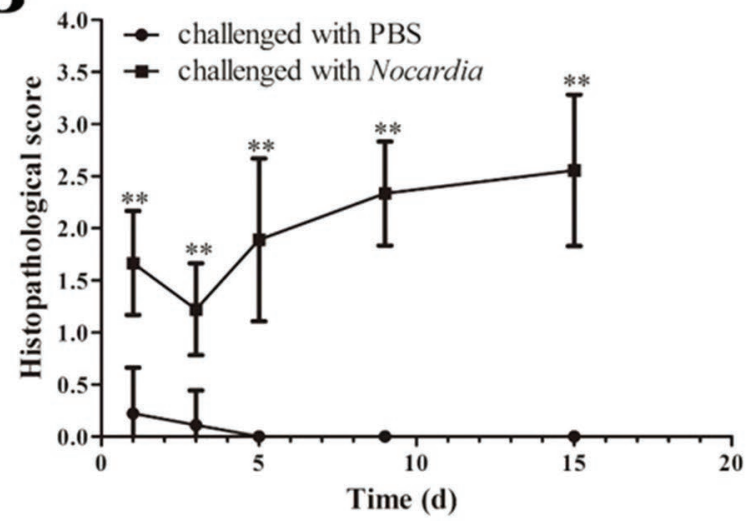

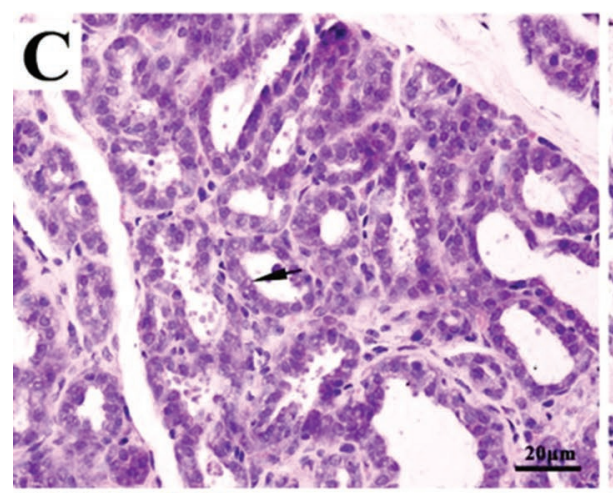
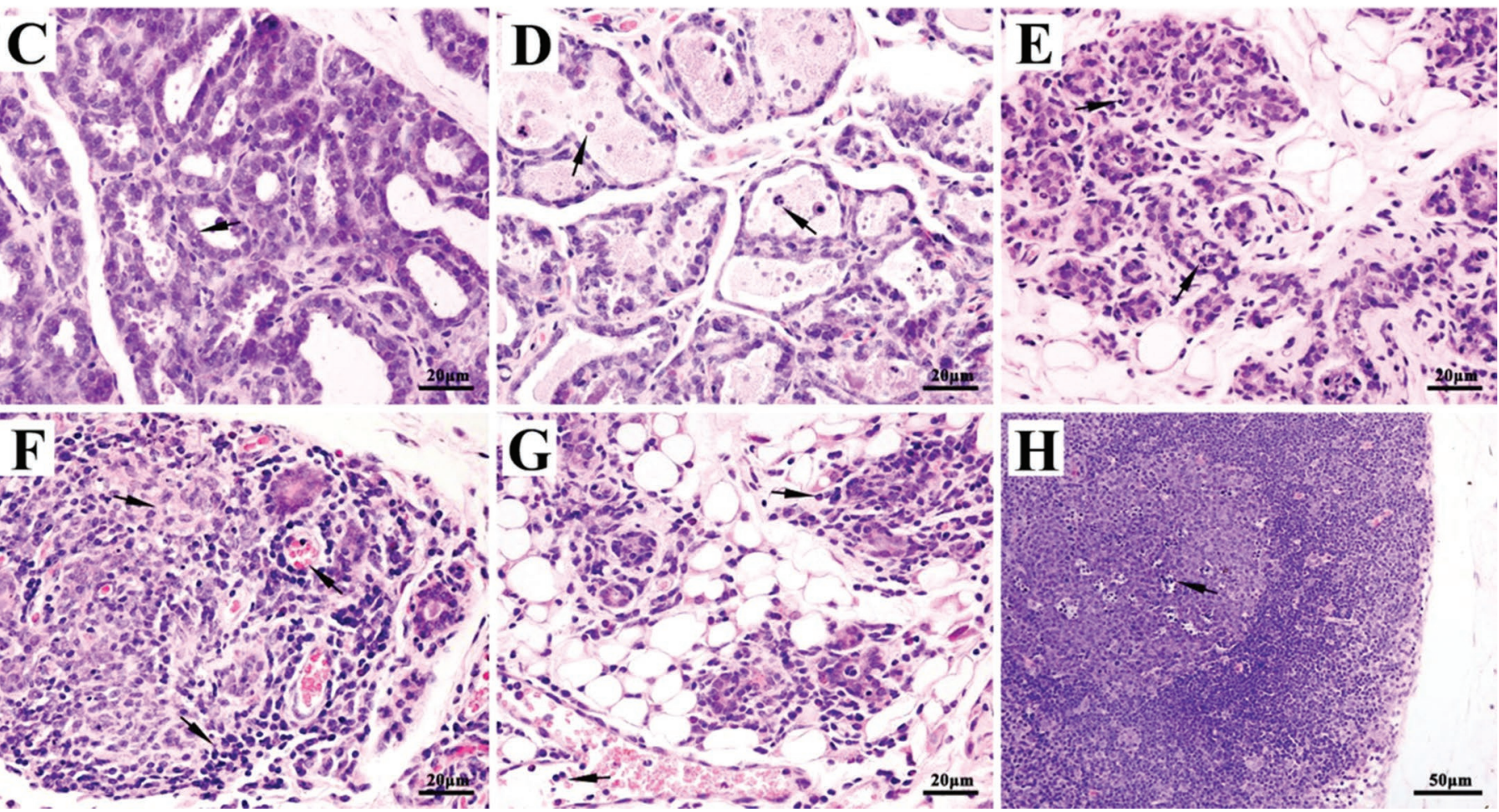

Figure 2. Clinical scores (A), histopathological scores (B), and histopathology of mammary tissues of mice challenged with clinical Nocardia cyriacigeorgica isolates (hematoxylin-eosin staining; C to H). Swollen mammary epithelial cells with vacuolated degeneration on d 1 of infection (C). Severe exfoliation appeared in the mammary alveoli on d 3 (D). Multiple inflammatory cells (black arrow) in interstitial and necrotic cells in atrophic alveoli on d 5 (E). Interstitial edema and lymphoid tissue in the mammary gland including lymphocytes, neutrophils, granulocytes, macrophages, and necrotic cells, on d 9 (F). Large numbers of inflammatory cells (black arrow) in mammary tissues and blood vessels on d 15 (G). Lymph node with dispersed necrotic areas in the germinal center on $\mathrm{d} 15(\mathrm{H})$. Results are presented as mean $\pm \mathrm{SD}$. $* * P<0.01$ was considered highly significant. Color version available online. 
we concluded that the $N$. cyriacigeorgica isolated likely originated from the environment without cross-infection among cows. Perhaps bedding or the water used at milking (or both) were contaminated with $N$. cyriacigeorgica, as cows with this pathogen contaminate the soil, contributing to transmission (Pisoni et al., 2008). That the bedding was very dirty and not frequently removed may have caused repeated challenge of cows with this pathogen (Saubolle and Sussland, 2003). Furthermore, the herd did not use self-compounded drugs that could have been contaminated with Nocardia, and it is therefore unlikely that this was the source of the infection.

Nocardia cyriacigeorgica, N. asteroides, and N. abscessus used to be classified as $N$. asteroides complex (Brown-Elliott et al., 2006). Moreover, N. cyriacigeorgica used to be known as $N$. asteroides (Brown-Elliott et al., 2015). The $16 \mathrm{~S}$ rRNA gene sequencing of $N$. cyriacigeorgica isolates had a homology of 98 to $99 \%$ to $N$. asteroides and N. abscessus, and all 3 had a close relationship in the phylogenetic tree. Although molecular methods, particularly 16S rRNA gene sequencing, are frequently used for taxonomy and identification of Nocardia spp. (Conville and Witebsky, 2007; Pisoni et al., 2008; Brown-Elliott et al., 2015), as done in the present study, biochemical characteristics and antimicrobial susceptibility patterns can also be used to verify the species.

The antibiotic susceptibility pattern of the clinical $N$. cyriacigeorgica isolates was type VI (resistant to ampicillin, amoxicillin-clavulanic acid, clarithromycin, and ciprofloxacin and susceptible to cephalosporins, amikacin, linezolid, and imipenem). This pattern is identical to the drug pattern of $N$. asteroides, whereas $N$. abscessus is reported to have a type I pattern (susceptible to ampicillin and amoxicillin-clavulanic acid; Brown-Elliott et al., 2006). Because $N$. cyriacigeorgica isolates were susceptible to amikacin, cefotaxime, ceftiofur, sulphamethoxazole, and imipenem, some could theoretically be used to treat mastitis caused by this pathogen, if diagnosed early. Due to the prolonged treatment with the low cure rate, human food safety, and public health concerns associated with drug residues, cattle suffering from nocardial mastitis should preferably be culled, which will also decrease the spread of nocardial infection (Cook and Holliman, 2004; Radostits et al., 2007).

Inoculation with clinical $N$. cyriacigeorgica isolates caused chronic inflammation of murine mammary glands. In addition, these mice had systemic symptoms and the contralateral mammary gland also became infected, implying that Nocardia may spread via blood circulation. This was apparently the first report to determine pathogenicity and histopathology of clinical
N. cyriacigeorgica isolates from bovine mastitis. Furthermore, very few studies reported histopathology of mammary tissues infected by other Nocardia spp. (Pisoni et al., 2008). In concordance to our study, Pisoni et al. (2008) also detected necrosis, infiltration of neutrophils and lymphocytes, and eosinophilic colonies of Nocardia-like organisms in bovine mammary tissues infected by $N$. neocaledoniensis during an outbreak.

To the best of our knowledge, this is the first report on the isolation, biochemical characteristics, and phylogenetic relatedness using $16 \mathrm{~S}$ rRNA gene sequencing and antimicrobial susceptibility patterns of $N$. cyriacigeorgica isolated from an outbreak of $\mathrm{CM}$ in a dairy herd. The clinical $N$. cyriacigeorgica isolates caused considerable damage to murine mammary glands, indicating that $N$. cyriacigeorgica has potential to cause bovine mastitis.

\section{ACKNOWLEDGMENTS}

This research was supported by the National Natural Science Foundation of China (no. 31572587 and no. 3151101034) and High-End Foreign Experts Recruitment Program (no. GDT20171100013). We are grateful for technical support from the Department of Electron Microscope and the Experimental Animal Centre of China Agricultural University. Additionally, we thank the Histopathology Laboratory of the College of Veterinary Medicine, China Agricultural University, for histopathological assessments.

\section{REFERENCES}

Ali, T., A. Rahman, M. S. Qureshi, M. T. Hussain, M. S. Khan, S. Uddin, M. Iqbal, and B. Han. 2014. Effect of management practices and animal age on incidence of mastitis in Nili Ravi buffaloes. Trop. Anim. Health Prod. 46:1279-1285.

Brown-Elliott, B. A., J. M. Brown, P. S. Conville, and R. J. Wallace. 2006. Clinical and laboratory features of the Nocardia spp. based on current molecular taxonomy. Clin. Microbiol. Rev. 19:259-282.

Brown-Elliott, B. A., P. Conville, and R. J. Wallace. 2015. Current status of Nocardia taxonomy and recommended identification methods. Clin. Microbiol. Newsl. 37:25-32.

Clinical and Laboratory Standards Institute. 2011. Susceptibility testing of mycobacteria, Nocardia, and other aerobic actinomycetes; approved standard, 2nd ed. (M24-A2). CLSI, Wayne, PA.

Clinical and Laboratory Standards Institute. 2012. Methods for dilution antimicrobial susceptibility tests for bacteria that grow aerobically; approved standard, 9th ed. (M07-A9). CLSI, Wayne, PA.

Clinical and Laboratory Standards Institute. 2013. Performance standards for antimicrobial disk and dilution susceptibility test for bacteria isolated from animals; approved standard, 4th ed. (VET01). CLSI, Wayne, PA.

Condas, L. A. Z., M. G. Ribeiro, K. Yazawa, A. P. C. de Vargas, T. Salerno, R. Giuffrida, H. Langoni, P. A. Melville, S. Biesdorf, T. Matsuzawa, T. Gonoi, J. P. Kastelic, and H. W. Barkema. 2013. Molecular identification and antimicrobial susceptibility of Nocar dia spp. isolated from bovine mastitis in Brazil. Vet. Microbiol. 167:708-712. 
Conville, P. S., and F. G. Witebsky. 2007. Organisms designated as Nocardia asteroides drug pattern type VI are members of the species Nocardia cyriacigeorgica. J. Clin. Microbiol. 45:2257-2259.

Cook, J. G., and A. Holliman. 2004. Mastitis due to Nocardia asteroides in a UK dairy herd following restocking after FMD. Vet. Rec. 154:267-268.

Gordon, R. E., D. A. Barnett, J. E. Handerhan, and C. N. Pang. 1974. Nocardia coeliaca, Nocardia autotrophica, and the nocardin strain. Int. J. Syst. Evol. Microbiol. 24:54-63.

Hashemi-Shahraki, A., P. Heidarieh, S. Z. Bostanabad, M. Hashemzadeh, M. M. Feiabadi, D. Schraufnagel, and M. Mirsaeidi. 2015. Genetic diversity and antimicrobial susceptibility of Nocardia species among patients with nocardiosis. Sci. Rep. 5:17862.

Kogure, T., R. Shimada, J. Ishikawa, K. Yazawa, J. M. Brown, Y. Mikami, and T. Gonoi. 2010. Homozygous triplicate mutations in three 16S rRNA genes responsible for high-level aminoglycoside resistance in Nocardia farcinica clinical isolates from a Canadawide bovine mastitis epizootic. Antimicrob. Agents Chemother. 54:2385-2390.

Liu, G., Y. Liu, T. Ali, M. Ferreri, J. Gao, W. Chen, J. Yin, J. Su, S. Fanning, and B. Han. 2015. Molecular and phenotypic characterization of Aerococcus viridans associated with subclinical bovine mastitis. PLoS One 10:e0125001.

Ollis, G. W., M. Schoonderwoerd, and C. Schipper. 1991. An investigation of risk factors for nocardial mastitis in central Alberta dairy herds. Can. Vet. J. 32:227-231.
Pisoni, G., C. Locatelli, L. Alborali, C. Rosignoli, S. Allodi, P. Riccaboni, V. Grieco, and P. Moroni. 2008. Short communication: Outbreak of Nocardia neocaledoniensis mastitis in an Italian dairy herd. J. Dairy Sci. 91:136-139.

Radostits, O. M., C. C. Gay, K. W. Hinchcliff, and P. D. Constable. 2007. Veterinary Medicine: A Text Book of the Diseases of Cattle, Sheep, Goats, Pigs and Horses. 10th ed. Bailliere Tindall, London, UK

Ribeiro, M. G., T. Salerno, A. L. D. Mattos-Guaraldi, T. C. F. Camello, H. Langoni, A. K. Siqueira, A. C. Paes, M. C. Fernandes, and G. H. B. Lara. 2008. Nocardiosis: An overview and additional report of 28 cases in cattle and dogs. Rev. Inst. Med. Trop. Sao Paulo 50:177-185.

Saubolle, M. A., and D. Sussland. 2003. Nocardiosis: Review of clinical and laboratory experience. J. Clin. Microbiol. 41:4497-4501.

Trivedi, M. 2015. Evaluation of antibiogram, genotype and phylogenetic analysis of biofield treated Nocardia otitidis. Biol. Syst. Open Access 4:143.

Xiao, M., L. Pang, S. C. Chen, X. Fan, L. Zhang, H. X. Li, X. Hou, J. Cheng, F. Kong, and Y. P. Zhao. 2016. Accurate identification of common pathogenic Nocardia species: Evaluation of a multilocus sequence analysis platform and matrix-assisted laser desorption ionization-time of flight mass spectrometry. PLoS One 11:e0147487. 